\title{
Development of sepsis in relation to serum biomarkers concentration like intercellular adhesion molecule-1, macrophage migration inhibitory factor, plasminogen activator inhibitor-1 and soluble Fas ligand
}

\begin{abstract}
Sepsis is a condition with high mortality rates and its diagnosis remains a challenge. Therefore, a new diagnostic biomarker or combinations of biomarkers for diagnosis of sepsis should be developed. In total, 80 patients who were suspected of having sepsis were followed up for 7 days. Quick Sepsis-related Organ Failure Assessment (qSOFA) scores were calculated day 1 and day 7; four biomarkers (intercellular adhesion molecule-1, macrophage migration inhibitory factor, plasminogen activator inhibitor-1 and soluble Fas ligand) results were evaluated the same. In 7 days in sepsis patients, blood serum concentrations of biomarker intercellular adhesion molecule-1 (ICAM-1) increased, $p=0.022$.
\end{abstract}

Keywords: sepsis, intercellular adhesion molecule-1, macrophage migration inhibitory factor, plasminogen activator inhibitor-1

\section{Introduction}

Sepsis is a complex and life-threatening systemic disease, with various of etiological factors and wide spectrum of severity [1]. A biomarker is a characteristic by which a (patho) physiologic process can be identified. Biomarkers can be of diagnostic value to discriminate infection from noninfectious conditions or to determine the causative pathogen, of prognostic value (assigning risk profiles and predict outcome), and in the future may be aid in selection and monitoring of therapy $[2,3]$.

We assessed epidemiological, clinical data and multiple biomarker profiles with respect to sepsis diagnosis and predictors of outcome. In the last 10 years almost 5000 articles on biomarkers in sepsis have been published $[4,5]$.

Inflammatory mediators, cell adhesion molecules (Intercellular Adhesion Molecule-1/ ICAM-1), redox active substances macrophage Migration Inhibitory Factor/MIF), plasminogen activator inhibitor-1 and soluble Fas ligand must be considered to be central hubs in the inflammatory process. However, their exact pathophysiologic function and prognostic value are still poorly understood. It is well known that endothelial activation is crucial in sepsis. [6,7].

Aim

To determine if serum levels of endothelial adhesion molecule 1 (ICAM-1), macrophage Migration Inhibitory Factor (MIF), Plasminogen Activator Inhibitor-1 (PAI-1) and Soluble Fas Ligand (sFAS) were associated with the development of severe clinical course in adult patients with sepsis.

\section{Material and Methods}

\section{Study population}

Patients were enrolled from October 2017 through April 2019 in the Emergency Department at Pauls Stradinš̌ Clinical University Hospital in Riga, Latvia.

Explicit criteria for enrollment included 1) age $>18$ years; 2) suspected or confirmed infection; 3) two or more systemic inflammatory response syndrome criteria.
Linda Bara ${ }^{1 *}$, Jelena Eglite $^{2}$, Peteris Oss ${ }^{3}$, Vinita Cauce ${ }^{4}$, Elvira Hagina ${ }^{2}$, Sandra Gintere ${ }^{1}$, Ludmila Viksna ${ }^{5}$ and Angelika Krumina ${ }^{5}$

${ }^{1}$ Department of Family Medicine, Riga Stradins University, Latvia ${ }^{2}$ Joint Laboratory of Clinical Immunology and Immunogenetics (JLCII), Riga Stradins University, Latvia ${ }^{3}$ Intensive Care and Reanimation Unit, Pauls Stradins Clinical University Hospital, Latvia

${ }^{4}$ Departmen of Physics, Riga Stradins University, Latvia ${ }^{5}$ Department of Infectology and Dermatology, Riga Stradins University, Latvia

*Author for correspondence:

linda.bridina@gmail.com 
Exclusion criteria included 1) age $<18$ years; 2) need for immediate surgery; and 3) absolute contraindication for a chest central venous catheter.

This study was approved by the Riga Stradins University Research Committee and Pauls Stradins Clinical University Hospital Research Committee and it met criteria for exemption from informed consent.

\section{- Data collection}

The hospitalization course of all patients was followed from admission to discharge. Using a standardized data collection instrument, data were collected on patients. The worst physiological and laboratory measurements within the initial $24 \mathrm{~h}$ of admission and second time after 7 days, were noted. The endpoint, all-cause in hospital mortality, was determined by systematically reviewing hospital records resulting from each patient's index hospitalization.

Severity of illness was estimated using the quick Sequential Organ Failure Assessment (qSOFA) score $[8,9]$. This score was calculated at Emergency Deparment (ED) recognition (day 1) and 7 days after intensive care unit admission. The primary outcome was in-hospital mortality.

\section{- Measurement of intercellular adhesion molecule-1, macrophage migration inhibitory factor, plasminogen activator inhibitor-1 and soluble Fas ligand}

Blood samples from septic patients were collected within $24 \mathrm{~h}$ after the time of sepsis diagnosis and second time after 7 days. In all patients we measured two times plasma levels ( $1^{\text {st }}$ day and $7^{\text {th }}$ day) of sICAM-1, MIF, sFAS ligand and tPAI-1 using reagent kit-Merck Millipore, HCYTOMAG-60K-04.

In Latvia these biomarkers such as ICAM1, MIF, PAI-1 and sFAS have not been studied previously, the authors chose these biomarkers because, given the pathophysiological mechanisms involved, the concentration of these biomarkers should increase in patients with sepsis.

\section{Statistical analysis}

The resulting study data were entered into an electronic database (Microsoft Excel 2010) and evaluated using SPSS software (Statistical
Package for the Social Sciences, 22.0 version, SPSS).

\section{Ethical approval}

This study was approved by the institutional review board of Riga Stradins University and Pauls Stadins Clinical University Hospital.

\section{Results}

\section{- Patient characteristics and outcome}

During the study period 80 patients fulfilled the inclusion criteria with ages ranging from 18 to 90 years. Patients characteristics and outcomes are shown in TABLE 1.

\section{Comparison of correlation of biomarkers and qSOFA}

All sepsis patients $n=80$ (100\%) within 7 days of hospitalization in intensive care unit, statistically significant increase in score after quick SOFA scale, $p=0.002$. After $q S O F A$ score 1 point in 1 st day were $n=48$ patients $(60 \%)$, 2 points were $\mathrm{n}=27(33.8 \%)$ and 3 points were $n=5$ patients $(6.3 \%)$. But after 7 days of hospitalization in intensive care unit, 1 point were $n=20(25 \%), 2$ points were $n=43(53.8 \%)$ and 3 points were $n=17(21.3 \%)$. The results also showed that mortality was higher in patients with higher qSOFA scores.

We also examined the correlation between 4 biomarkers (intercellular adhesion molecule-1, macrophage migration inhibitory factor, plasminogen activator inhibitor- 1 and soluble Fas ligand) levels in 1st day after hospitalization in intensive care unit and second time after 7 days. Sepsis patients showed ICAM-1 concentration on day 1 was Me 12245.347 (Q1 6228.500; Q3 33372.419) pg/ml, but on day 7 Me 11107.250 (Q1 4774.00; Q3 30356.503) $\mathrm{pg} / \mathrm{ml}, \mathrm{p}=0.022$. The statistician did not change the biomarkers MIF and PAI-1 significantly, on day 1 MIF was Me 93.266 (Q1 47.750; Q3 177.669) $\mathrm{pg} / \mathrm{ml}$, but on day $7 \mathrm{Me} 99.004$ (Q1 45.000; Q3 201.723) pg/ml, $\mathrm{p}=0.580$. PAI-1 concentration on day 1 was Me 5857.250 (Q1 3928.511; Q3 9589.500) pg/ml, on day 7 Me 6222.208 (Q1 4003.950; Q3 8553.728) $\mathrm{pg} / \mathrm{ml}, \mathrm{p}=0.337$ and no statistically significant association was found between day 1 and day 7 of the sFAS ligand biomarker, respectively, sFAS ligand concentration on day 1 was Me 2321.105 (Q1 1023.006; Q3 6471.201) pg/ml, on day 7 Me 3304.281 (Q1 2466.098; Q3 7698.245) $\mathrm{pg} / \mathrm{ml}, \mathrm{p}=0.167$. 


\begin{tabular}{|c|c|c|c|c|}
\hline \multirow[t]{2}{*}{ Variables } & Total & Survivor & Nonsurvivor & \multirow[t]{2}{*}{ P-value } \\
\hline & $(n=80 ; 100 \%)$ & $(n=65 ; 81.3 \%)$ & $(n=15 ; 18.8 \%)$ & \\
\hline Sex (Male) & $51(63.8 \%)$ & $42(64.6 \%)$ & $9(60.0 \%)$ & \multirow[t]{2}{*}{0.727} \\
\hline Sex (Female) & $29(36.3 \%)$ & $23(35.4 \%)$ & $6(40.0 \%)$ & \\
\hline \multicolumn{5}{|c|}{ Age } \\
\hline $18-40$ years & $8(10.0 \%)$ & $7(10.8 \%)$ & $1(6.7 \%)$ & \multirow[t]{3}{*}{0.829} \\
\hline $41-60$ years & $26(32.5 \%)$ & $21(32.3 \%)$ & $5(33.3 \%)$ & \\
\hline $61-90$ years & $46(57.5 \%)$ & $37(56.9 \%)$ & $9(60.0 \%)$ & \\
\hline \multicolumn{5}{|c|}{ Site of primary infection } \\
\hline Lower respiratory tract infection & $37(46.3 \%)$ & $30(46.2 \%)$ & $7(46.7 \%)$ & \multirow[t]{5}{*}{0.396} \\
\hline Intra-abdomen infection & $16(20 \%)$ & $12(18.5 \%)$ & $4(26.7 \%)$ & \\
\hline Skin and musculoskeletal infection & $17(21.3 \%)$ & $16(24.6 \%)$ & $1(6.7 \%)$ & \\
\hline Urinary tract infection & $6(7.5 \%)$ & $4(6.2 \%)$ & $2(13.7 \%)$ & \\
\hline Unknown etiology & $4(5.0 \%)$ & $3(4.6 \%)$ & $1(6.7 \%)$ & \\
\hline \multicolumn{5}{|c|}{ Organ dysfunction } \\
\hline Acute respiratory failure & $22(27.5 \%)$ & $15(44.1 \%)$ & $7(58.3 \%)$ & \multirow[t]{4}{*}{0.262} \\
\hline Acute renal failure & $8(10.0 \%)$ & $6(23.5 \%)$ & $0(0.0 \%)$ & \\
\hline Septic shock & $11(13.8 \%)$ & $7(20.6 \%)$ & $4(33.3 \%)$ & \\
\hline Altered consciousness & $5(6.3 \%)$ & $4(11.8 \%)$ & $1(8.3 \%)$ & \\
\hline \multicolumn{5}{|c|}{ Comorbidities } \\
\hline Pulmonary disease & $17(21.3 \%)$ & $11(21.6 \%)$ & $6(42.9 \%)$ & \multirow[t]{7}{*}{0.209} \\
\hline Cardiovascular disease & $22(27.5 \%)$ & $18(35.3 \%)$ & $4(28.6 \%)$ & \\
\hline Malignancies & $6(7.5 \%)$ & $5(9.8 \%)$ & $1(7.1 \%)$ & \\
\hline Diabetes mellitus & $9(11.3 \%)$ & $9(17.6 \%)$ & $0(0.0 \%)$ & \\
\hline Chronic renal disease & $4(5.0 \%)$ & $2(3.9 \%)$ & $2(14.3 \%)$ & \\
\hline Immune deficiency & $3(3.8 \%)$ & $2(3.9 \%)$ & $1(7.1 \%)$ & \\
\hline Other & $4(5.0 \%)$ & $4(7.8 \%)$ & $0(0.0 \%)$ & \\
\hline \multicolumn{5}{|c|}{ Severity score (qSOFA* ) at day 1} \\
\hline qSOFA=1 & $48(60.0 \%)$ & $38(58.5 \%)$ & $10(66.7 \%)$ & \multirow[t]{3}{*}{0.002} \\
\hline$q S O F A=2$ & $27(33.8 \%)$ & $22(33.8 \%)$ & $5(33.3 \%)$ & \\
\hline$q S O F A=3$ & $5(6.3 \%)$ & $5(7.7 \%)$ & $0(0.0 \%)$ & \\
\hline \multicolumn{5}{|c|}{ Severity score (qSOFA* ) at day 7} \\
\hline$q S O F A=1$ & $20(25.0 \%)$ & $20(30.8 \%)$ & $0(0.0 \%)$ & \multirow[t]{3}{*}{$<0.001$} \\
\hline$q S O F A=2$ & $43(53.8 \%)$ & $40(61.5 \%)$ & $3(20.0 \%)$ & \\
\hline$q S O F A=3$ & $17(21.3 \%)$ & $5(7.7 \%)$ & $12(80.0 \%)$ & \\
\hline
\end{tabular}

FIGURE 1. Intercellular adhesion molecule-1 (ICAM-1) concentration at $1^{\text {st }}$ day after hospitalization in intensive care unit and at $7^{\text {th }}$ day. ICAM-1 concentration level in sepsis patient's blood samples $(\mathrm{pg} / \mathrm{ml})$.

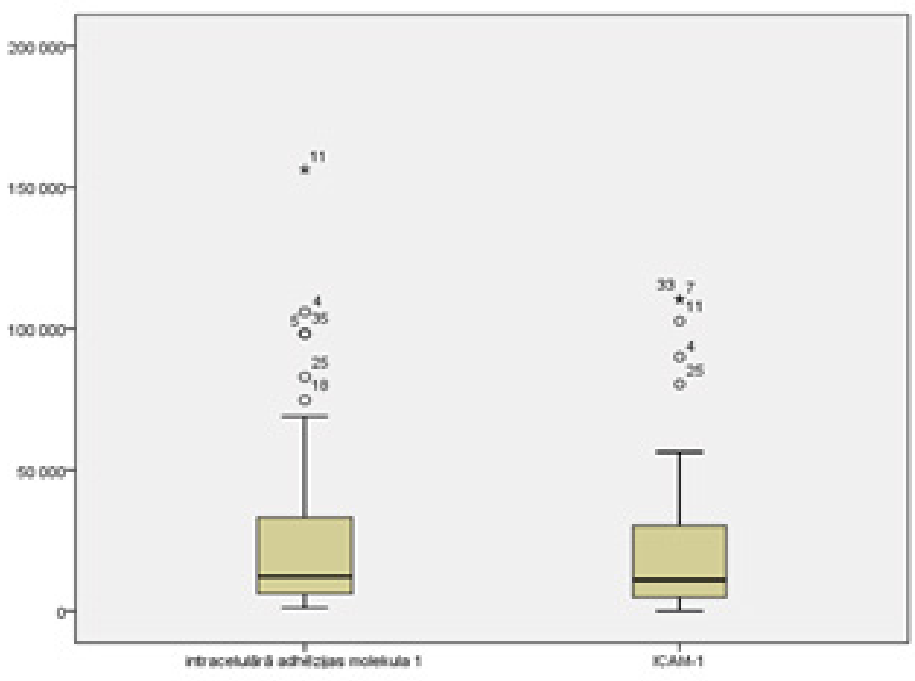

1st day in intensive care unit $\quad 7$ th day in intensive care unit 


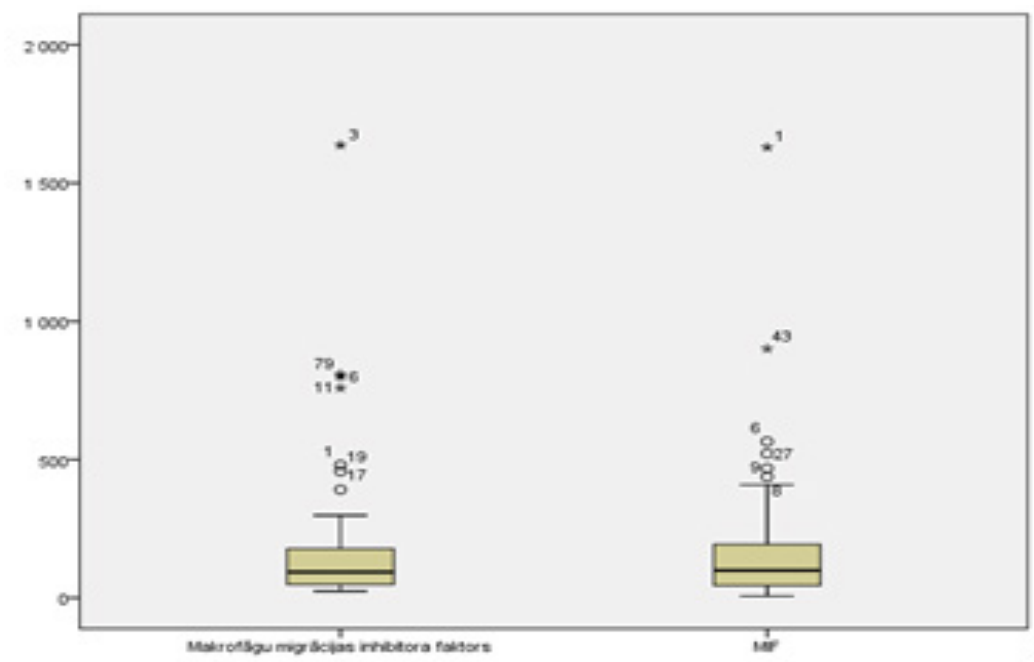

1 st day in intensive care unit

7th day in intensive care unit

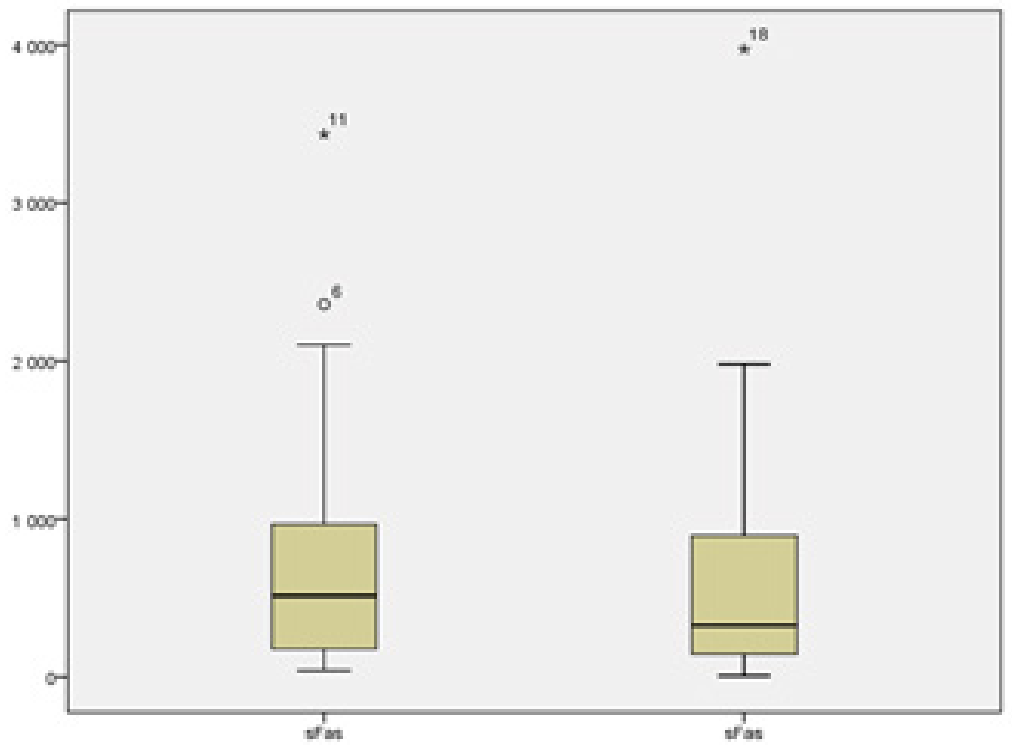

1st day in intensive care unit 7 th day in intensive care unit
FIGURE 2. Macrophage migration inhibitory factor (MIF) concentration at $1^{\text {st }}$ day after hospitalization in intensive care unit and at $7^{\text {th }}$ day. MIF concentration level in sepsis patient's blood samples (pg/ml).

FIGURE 3. Soluble Fas ligand at $\mathbf{1}^{\text {st }}$ day after hospitalization in intensive care unit and at $7^{\text {th }}$ day. Solubale FAS ligand concentration level in sepsis patient's blood samples (pg/ml).
On Day 1 and the biomarker $t$ PAI-1, correlation coefficient was $r=0.572, p<0.001$ as well as day $7 \mathrm{r}=0.360, \mathrm{p}=0.001$. MIF on day 1 $\mathrm{r}=0.131, \mathrm{p}=0.246$, on day $7 \mathrm{r}=0.122, \mathrm{p}=0.281$. ICAM- 1 on day 1 was $r=0.078, p=0.492$, but on day $7 \mathrm{r}=0.102, \mathrm{p}=0.368$. And about biomarker sFAS ligand on day 1 , correlation coefficient was $\mathrm{r}=0.126, \mathrm{p}=0.265$, on day $7 \mathrm{r}=0.062, \mathrm{p}=0.585$.

The box plots of the 4 biomarkers stratified by concentration level in blood samples are showns in FIGURES 1-4.

The results also showed that all patients who died had higher levels of biomarkers than those who survived, this was particularly significant for the biomarker tPAI-1 $(\mathrm{p}=0.017)$. All results are shown in TABLE 2.

\section{Discussion}

This study focused on clinical-epidemiological and biomarkers profiles of patients admitted to the public hospital in Pauls Stradiņš Clinical University Hospital in Riga, Latvia for a period of 3 years. Regarding the epidemiological characteristics, predominance of male was in agreement with that reported in previous worldwide studies, [10-12] and the median age was the same than that observed in most centers and private hospitals in Europe. 
FIGURE 4. Plasminogen activator inhibitor-1 (tPAI-1) concentration at $1^{\text {st }}$ day after hospitalization in intensive care unit and at $7^{\text {th }}$ day. tPAI- 1 concentration level in sepsis patient's blood samples $(\mathrm{pg} / \mathrm{ml})$.

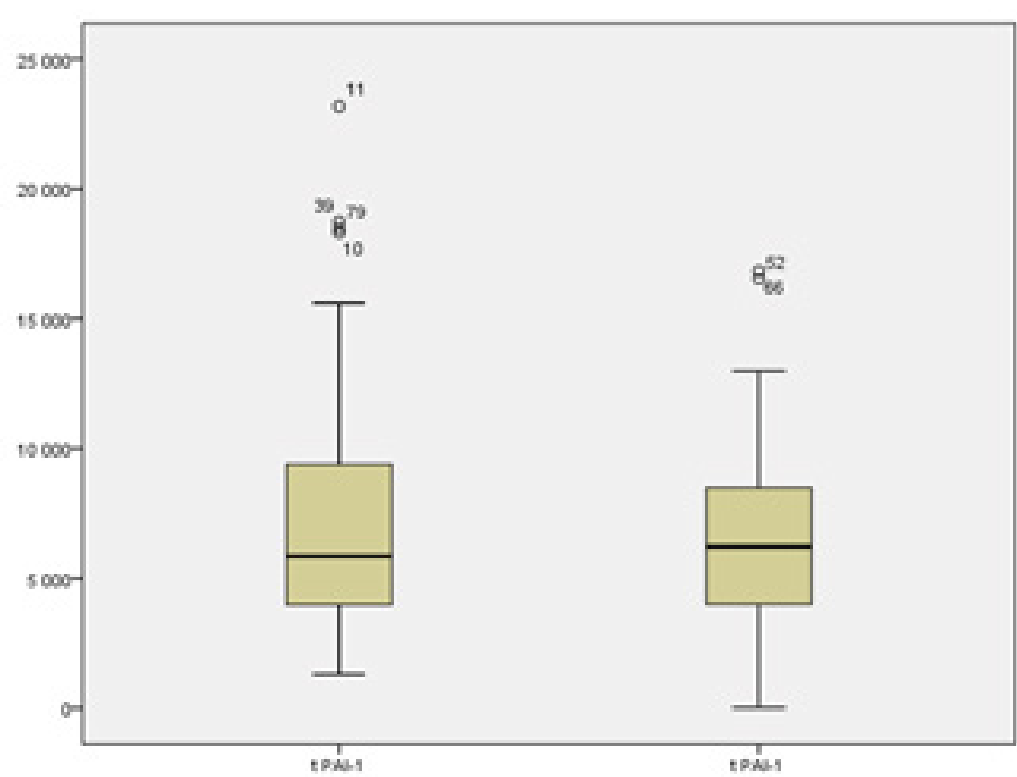

1 st day in intensive care unit 7 th day in intensive care unit

\begin{tabular}{|c|c|c|c|c|}
\hline Biomarkers & Patients outcome & N (number; \%) & Mean Rank (Me) pg/ml & P-value \\
\hline \multirow[t]{2}{*}{$\mathrm{MIF}^{*}$} & Survivor & $65(81.3 \%)$ & 39.63 & \multirow[t]{2}{*}{0.486} \\
\hline & Nonsurvivor & $15(18.8 \%)$ & 44.27 & \\
\hline \multirow[t]{2}{*}{ ICAM-1* } & Survivor & $65(81.3 \%)$ & 40.48 & \multirow[t]{2}{*}{0.985} \\
\hline & Nonsurvivor & $15(18.8 \%)$ & 40.6 & \\
\hline \multirow[t]{2}{*}{ Sfas $^{*}$} & Survivor & $65(81.3 \%)$ & 40.15 & \multirow[t]{2}{*}{0.777} \\
\hline & Nonsurvivor & $15(18.8 \%)$ & 42.03 & \\
\hline \multirow[t]{2}{*}{ tPAI-1* } & Survivor & $65(81.3 \%)$ & 37.52 & \multirow[t]{2}{*}{0.017} \\
\hline & Nonsurvivor & $15(18.8 \%)$ & 53.4 & \\
\hline \multicolumn{5}{|c|}{ *MIF-Macrophage Migration Inhibitory Factor } \\
\hline \multicolumn{5}{|c|}{ *ICAM-1-Intercellular Adhesion Molecule-1 } \\
\hline \multicolumn{5}{|c|}{ "sFAS-Soluble Fas Ligand } \\
\hline \multicolumn{5}{|c|}{${ }^{*}$ tPAI-1-Plasminogen Activator Inhibitor-1 } \\
\hline
\end{tabular}

The presence or absence of comorbidities influence the outcome, and association was found between sepsis severity and baseline disease. These data are similar when analyzing other epidemiological studies.

Intercellular Adhesion Molecule-1 (ICAM1), mediating adherence between cells and extracellular matrix, could release in cascade to damage the vascular endothelial cells during sepsis [7]. ICAM-1 has been investigated in sepsis. To date, many studies showed that its level is increased in patients with sepsis [1315]. Bavunoglu et al. found that ICAM-1 had a high sensitivity (99\%) and specificity (99\%) for detecting sepsis. Thus, ICAM-1 might be an effective biomarker for diagnosing sepsis [1619].
Our study also showed that ICAM-1 levels increase with the clinical severity of sepsis. High levels of adhesion molecules have been found in sepsis patients. We found that, in 7 days in sepsis patients, blood serum concentrations of biomarker ICAM-1 increased, $\mathrm{p}=0.022$, what could be a significant biomarker for the evaluation of sepsis severity. Higher mortality was also observed in these patients. It is well known that fibrin removal is severely hindered by inactivation of the endogenous fibrinolytic system, mainly as a result of upregulation of Plasminogen Activator Inhibitor type-1 (PAI-1) $[8,9]$.

In pathogenesis of sepsis, PAI-1 plays a role in several biological processes dependent on the inhibition of plasminogen activators and plasmin 
activity. As a consequence, overproduction of PAI-1 may contribute to organ dysfunction in patients suffering from severe infection [20,21]. We demonstrated that serum $t$ PAI-1 also has better correlation with clinical severity (after $\mathrm{qSOFA}=3$ points) than biomarkers MIF and sFAS ligand). For example, those patients with initially high PAI-1 levels may be offered a more proactive management, including early ICU admission, initiation of fluid resuscitation, and inotropic support [21].

About MIF, our results showns that, there was no statistically significant relationship between the MIF and qSOFA scales, i.e., day $1 \mathrm{r}=0.131, \mathrm{p}=0.246$, day $7 \mathrm{r}=0.122, \mathrm{p}=0.281$. However, no sufficient knowledge exists about the prognostic value of these key mediators in severe sepsis $[22,23]$.

\section{Conclusion}

In 7 days in sepsis patients, blood serum concentrations of biomarker intercellular adhesion molecule-1 (ICAM-1) increased, $\mathrm{p}=0.022$, what could be a significant biomarker for the evaluation of sepsis severity. The results also showed that all patients who died had higher levels of biomarkers than those who survived, this was particularly significant for the biomarker tPAI-1 $(p=0.017)$. Therefore, the study would be important to continue and increase the number of patients included in the study that could change the statistical results. 


\section{REFERENCES}

Schmidt de Oliveira-Netto AC, Morello LG, Dalla-Costa LM, et al. Procalcitonin, c-reactive protein, albumin, and blood cultures as early markers of sepsis diagnosis or predictors of outcome: A prospective analysis. Clin Pathol. 12, $2632010 X 19847673$ (2019).

Li XJ, Tan EL, Zhao CP, et al. Accuracy of intercellular adhesion molecule-1 for diagnosing sepsis. A systematic review and meta-analysis protocol. Medicine (Baltimore). 98, e16019 (2019).

Van Engelen TSR, Wiersinga WJ, Scicluna BP, et al. Biomarkers in sepsis. Crit Care Clin. 34, 139-152 (2018).

J Han JE, Martin GS. Biomarkers in sepsis: On target or off the mark? Am J Med Sci. 354, 3-4 (2017).

Yu H, Nie L, Liu A, et al. Combining procalcitonin with the qSOFA and sepsis mortality prediction. Medicine (Baltimore). 98, e15981 (2019).

Xing LY, Yin J, Shao M, et al. Clinical characteristics and prognosis of serous body cavity effusions in patients with sepsis: a retrospective observational study. $B M C$ Anesthesiol. 18, 169 (2018).

Zonneveld R, Jongman RM, Juliana A, et al. Serum concentrations of endothelial cell adhesion molecules and their shedding enzymes and early onset sepsis in newborns in Suriname. BMJ Paediatr Open. 2, e000312 (2018).

Jones AE, Trzeciak S, Kline JA. The Sequential Organ Failure Assessment score for predicting outcome in patients with severe sepsis and evidence of hypoperfusion at the time of emergency department presentation. Crit Care Med. 37, 1649-1654 (2009).

Ferreira FL, Bota DP, Bross A, et al. Serial evaluation of the SOFA score to predict outcome in critically ill patients. JAMA. 286, 1754-1758 (2001).

Kaukonen KM, Bailey M, Suzuki S, et al. Mortality related to severe sepsis and septic shock among critically ill patients in Australia and New Zealand, 2000-2012. JAMA. 311, 1308-1316 (2014).

Martin GS, Mannino DM, Eaton S, et al. The epidemiology of sepsis in the United States from 1979 through 2000. N Engl J Med. 348, 1546-1554 (2003).

Vincent JL, Sakr Y, Sprung CL, et al. Sepsis occurrence in acutely ill patients investigators. Crit Care Med. 34, 344-353 (2006).

Li XJ, Tan EL, Zhao CP, et al. Accuracy of intercellular adhesion molecule-1 for diagnosing sepsis. A systematic review and meta-analysis protocol. Medicine (Baltimore). 98, e16019 (2019).

Larson RS, Springer TA. Structure and function of leukocyte integrins. Immunol Rev. 114, 181-217 (1990).

Gearing AJ, Newman W. Circulating adhesion molecules in disease. Immunol Today. 14, 506-512 (1993).

Freund Y, Lemachatti N, Krastinova E, et al. Prognostic accuracy of sepsis-3 criteria for in-hospital mortality among patients with suspected infection presenting to the emergency department. JAMA. 317, 301-308 (2017).

Kayal S, Jaïs JP, Aguini N, et al. Elevated circulating e-selectin, intercellular adhesion molecule 1 , and von Willebrand factor in patients with severe infection. Am J Respir Crit Care Med. 157, 776-784 (1998).

Veleminsky M, Stransky P, Veleminsky $\mathrm{M}$, et al. Relationship of IL-6, IL-8, TNF and sICAM-1 levels to PROM, pPROM, and the risk of early-onset neonatal sepsis. Neuro Endocrinol Lett. 29, 303-311 (2008).

Bavunoglu I, Genc H, Konukoglu $\mathrm{D}$, et al. Oxidative stress parameters and inflammatory and immune mediators as markers of the severity of sepsis. J Infect Dev Ctries. 10, 1045-1052 (2016).

Lijnen HR. Pleiotropic functions of plasminogen activator inhibitor-1.J Thromb Haemost. 3, 35-45 (2005).

Tipoe TL, Wu WKK, Chung L, et al. Plasminogen activator inhibitor 1 for predicting sepsis severity and mortality outcomes: A systematic review and metaanalysis. Front Immunol. 18, 1218 (2018).

Brenner T, Hofer S, Rosenhagen C, et al. Macrophage migration inhibitory factor (mif) and manganese superoxide dismutase (mnsod) as early predictors for survival in patients with severe sepsis or septic shock. J Surg Res. 164, e163-e171 (2010).

Al-Abed Y, Dabideen D, Aljabari B, et al. ISO-1 binding to the tautomerase active site of MIF inhibits its pro-inflammatory activity and increases survival in severe sepsis. J Biol Chem. 280, 36541-36544 (2005). 